\title{
Boundary Layer Profile Behind Gaseous Detonation as it Affects Reflected Shock Wave Bifurcation
}

\author{
J. Damazo, ${ }^{*}$ J. Odell, ${ }^{\dagger}$ and J. E. Shepherd ${ }^{\ddagger}$ \\ California Institute of Technology, Pasadena, CA, 91125, USA
}

\begin{abstract}
The present study explores the flow field created by reflecting detonations using heat transfer and pressure measurements near the location of detonation reflection. Schlieren imaging techniques are used to examine the possibility of shock wave-boundary layer interaction. These measurements are compared to laminar boundary layer theory and a onedimensional model of detonation reflection. Experiments were carried out in a $7.6 \mathrm{~m}$ long detonation tube with a rectangular test section using mixtures of stoichiometric hydrogenoxygen with argon dilution of $0,50,67$, and $83 \%$ at an initial pressure of 10,25 , and $40 \mathrm{kPa}$. Optical observations show that minimal interaction of the reflected shock wave results when propagating into the boundary layer created by the incident wave. The heat transfer rate is qualitatively consistent with the time dependent laminar boundary layer predictions, however the magnitude is consistently larger and substantial (factor of three) peak-to-peak fluctuations are observed. The pressure measurements show good agreement between predicted ideal incident and reflected wave speeds. The pressure amplitudes are under-predicted for no argon dilution cases particularly at $40 \mathrm{kPa}$, but in reasonable agreement for lower pressures and higher dilutions.
\end{abstract}

\section{Nomenclature}

Subscripts

$1 \quad$ Initial experimental conditions

2 Post-detonation (Chapman-Jouguet) conditions

3 Burned equilibrium conditions

Symbols

$\eta \quad$ Similarity variable used in the solution to the laminar boundary layer equations

$\gamma \quad$ Ratio of specific heats

$\nu \quad$ Kinematic viscosity

$c \quad$ Fluid sound speed

$c_{p} \quad$ Specific heat at constant pressure

$f \quad$ Non-dimensionalized velocity used in the similarity solution to the laminar boundary layer equations

$h \quad$ Enthalpy

$k \quad$ Fluid heat conductivity

$p \quad$ Pressure

$p_{R} \quad$ Pressure at the reflecting end-wall

$q \quad$ Heat transfer per unit area

$u \quad$ Fluid velocity

Re Reynolds number

${ }^{*} \mathrm{PhD}$ Candidate at the Graduate Aerospace Laboratories California Institute of Technology, 1200 E California Blvd, MC 105-50, Pasadena, CA, 91125. AIAA student member.

†Senior Mechanical Engineer at the California Institute of Technology, 1200 E California Blvd, MC 105-50, Pasadena, CA, 91125. AIAA student member.

${ }^{\ddagger}$ C.L. "Kelly" Johnson Professor of Aeronautics and Professor of Mechanical Engineering at the Graduate Aerospace Laboratories California Institute of Technology, 1200 E California Blvd, MC 105-50, Pasadena, CA, 91125. AIAA senior member. 
$R e_{1} \quad$ Reynolds number evaluated $1 \mathrm{~m}$ behind the detonation

St Stanton number

$T \quad$ Temperature

$U_{I} \quad$ Measured incident detonation speed

$U_{C J} \quad$ Theoretical Chapman-Jouguet detonation speed

\section{Introduction}

A Particularly important situation in laboratory testing or accidental explosions is the initiation of a Aflame followed by deflagration to detonation transition (DDT), resulting in a detonation propagating in a piping system. ${ }^{1}$ Gaseous detonations ${ }^{2,3}$ inside piping or vessels create both structural and thermal loads and in extreme cases may lead to plastic deformation or rupture. ${ }^{4}$ When the detonation reaches a closed end, the boundary condition of zero flow velocity leads to the creation of a reflected shock wave that propagates back towards the point of ignition. ${ }^{5}$ The speed and strength of this reflected shock wave changes with time as the shock propagates through the unsteady Taylor-Zel'dovich wave. ${ }^{6,7}$ The reflected shock wave is also complicated by the possibility of interaction with the boundary layer created by the flow induced by the incident gaseous detonation. ${ }^{8}$ Recent work in our laboratory by Karnesky et al., ${ }^{4,8}$ has suggested that the internal pressure and resulting plastic deformation of thin-walled cylindrical shells due to internal detonation loading is influenced by these viscous effects. There is evidence that the boundary layer created by the fluid motion induced by the detonation interacts with the reflected shock wave created when the detonation normally impinges upon a planar wall. The goal of this paper is to present analytical and experimental findings on the behavior and nature of the boundary layer induced by the detonation and its effects on the reflected shock wave created when a detonation normally impinges upon an end wall.

Reflected shock wave bifurcation occurs when a normally reflected shock wave splits into a lambda shock as shown in Figure 1 due to interaction with the boundary layer created by the incident shock. Previous researchers have extensively examined bifurcation in shock tubes as it pertains to shock tube performance. ${ }^{9-12}$ Mark developed the foundational theory that explains and predicts under what conditions bifurcation will occur. ${ }^{9}$ Mark shows that gases exhibiting a low ratio of specific heats, $\gamma$, bifurcate under the widest range of conditions; this suggests that reflected detonations, which have an especially low value for $\gamma$, will readily bifurcate and makes bifurcation a likely candidate for explaining the two-dimensional effects observed in our laboratory's previous work. However, Mark's analysis assumes the temperature is constant and equal to the initial conditions. This assumption is invalid for the detonation case and is one of the motivations for this study of the boundary layer behind a detonation and interaction with the reflected shock wave.

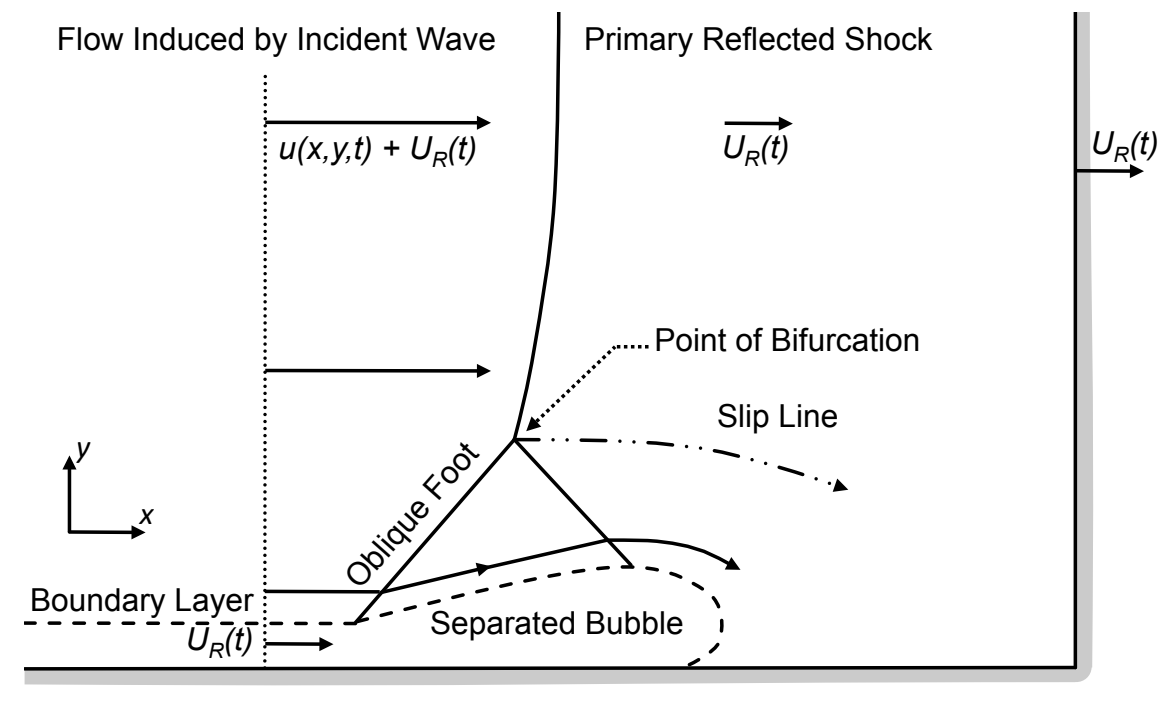

Figure 1. Archetypical reflected shock wave bifurcation sketched in the shock fixed frame.

Numerous researchers have studied boundary layer growth behind shock waves. Mirels ${ }^{13,14}$ developed much of the pioneering work on boundary layer growth behind shock waves. Sturtevant and Okamura ${ }^{15}$ 
solved the boundary layer equation in the shock-fixed frame and explored the effects of shock strength on the boundary layer profile. Liu et al. ${ }^{16}$ applied boundary layer analyses to the case of hemi-spherical blast and detonation waves. Here we adapt these models of boundary layer development behind shock waves to the case of boundary layer growth behind detonation. We compare the results to pressure and heat flux measurements recorded during gaseous detonation experiments of stoichiometric hydrogen-oxygen at varying initial pressure and varying argon dilution, conditions which result in varying free-stream conditions as well as detonation cell size. Alongside these measurements we present schlieren photographs of the incident detonation and reflected shock. These pictures reveal novel behavior of the shock wave where it is made up of multiple shock waves. The photographs further reveal the presence of shock wave-boundary layer interaction over a range of argon dilutions.

\section{Analytical Formulation}

One approach to modeling the detonation reflection process is numerical simulation of reacting viscous compressible flows. ${ }^{17-20}$ Previous studies on reflecting shock waves ${ }^{9,21}$ however, suggest that much may be gained in understanding the shock wave-boundary layer interaction by applying simple models in analyzing the boundary layer growth and propagation of the reflected shock wave. As a first step, we apply previous models for boundary layer growth and detonation reflection to the experimental cases of stoichiometric hydrogen-oxygen detonations with argon dilution. At the present stage, we are still developing an analytical model that includes the strong property variations within the boundary layer and the present experimental results serve to inform our efforts in that direction.

Although gaseous detonations are inherently three-dimensional due to their cellular structure, their mean properties are well approximated by one-dimensional wave propagation theory. ${ }^{22}$ This results in the detonation exhibiting the behavior sketched in the $x-t$ diagram shown in Figure 2. The detonation propagates

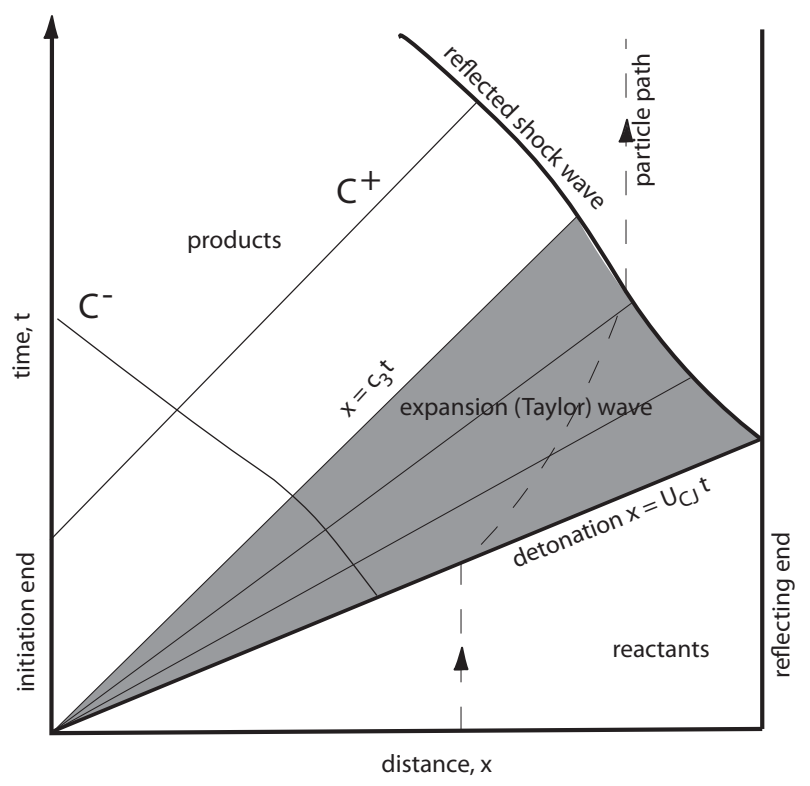

Figure 2. One-dimensional behavior of a detonation in a tube.

away from the point of ignition at the constant theoretical Chapman-Jouguet (CJ) velocity, $U_{C J}$, towards the closed end of the tube. The Taylor-Zel'dovich (TZ) expansion wave trails the detonation and gradually brings the fluid to rest. Once the detonation impinges upon the tube's closed end, a reflected shock wave propagates into the TZ expansion at a non-constant speed. Analysis of the speed and strength of this unsteady reflected wave is discussed in previous work. ${ }^{4}$ To simplify the analytical investigation of reflecting detonation waves, we will consider the less complicated case shown in Figure 3 where the TZ expansion wave is neglected and the properties behind the detonation are the constant CJ values. This assumption becomes 


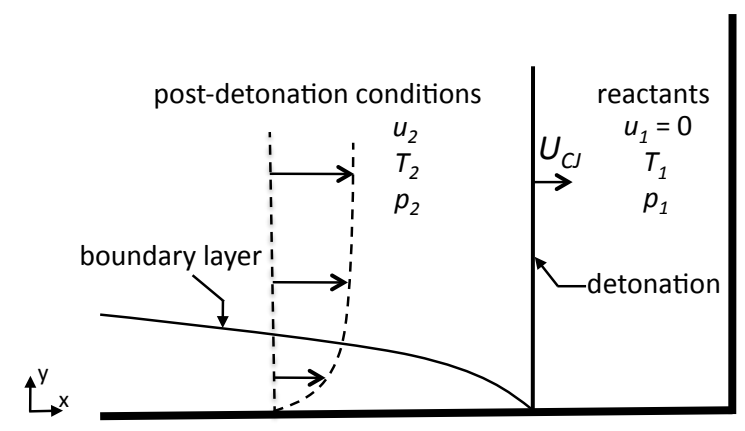

(a)

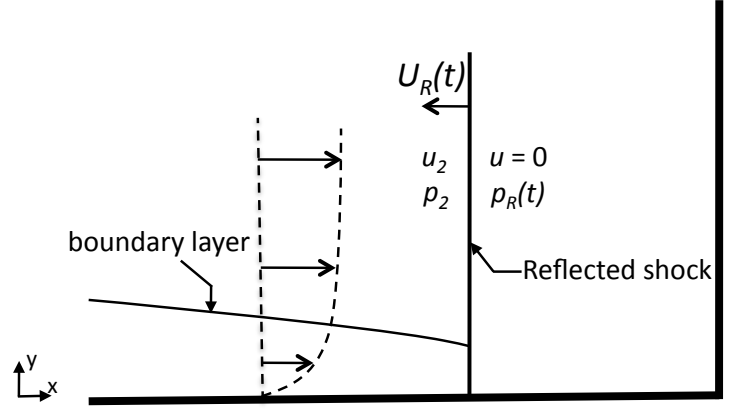

(b)

Figure 3. Relevant fluid dynamic and thermodynamic parameters involved in the detonation reflection process shown in the lab-fixed frame of reference used in all mathematical derivations presented herein sketched (a) before and (b) after detonation reflection occurs.

more accurate as the gradients in the $\mathrm{TZ}$ wave become smaller which occurs in long detonation tubes such as the $7.6 \mathrm{~m}$ long GALCIT Detonation Tube (GDT) discussed in Section III.

\section{A. Boundary Layer Analysis}

For the configuration shown in Figure 3, we can develop an approximate analytical laminar boundary layer solution to predict the boundary layer profile and heat transfer to the wall up until the arrival of the reflected shock wave. Let us suppose the detonation is initiated at $x=0, t=0$. In front of the detonation, $t>x / U_{C J}$, the gas is stationary at initial pressure $p_{1}$ and temperature $T_{1}$. Behind the detonation, $t<x / U_{C J}$, the free-stream properties are the computed Chapman-Jouguet post-detonation properties $p_{2}$ and $T_{2}$. The freestream properties (written with a sub-script 2) are used to define laminar boundary layer solution parameters and are calculated with the shock and detonation toolbox. ${ }^{23}$ Through the Levy-Lees transformation, ${ }^{24}$ the two-dimensional compressible laminar boundary layer equations can be transformed with the similarity variable $\eta$ where

$$
\eta(x, y, t)=\frac{1}{\delta(x, t)} \int_{0}^{y} \frac{\rho\left(x, y^{\prime}, t\right)}{\rho_{2}} d y^{\prime}
$$

and $\delta$ is the boundary layer thickness written in the lab-fixed frame

$$
\delta(x, t)=\sqrt{\nu_{2}\left(t-\frac{x}{U_{C J}}\right)} .
$$

Assuming that the detonation products behave as a perfect gas, the analysis results in an ordinary differential equation in $\eta$ which may be solved to yield the fluid velocity:

$$
0=f^{\prime \prime \prime}+\frac{1}{2}\left(\eta-\frac{u_{2}}{U_{C J}} f\right) f^{\prime \prime}
$$

using

$$
f(\eta)=\int_{0}^{\eta} \frac{u\left(\eta^{\prime}\right)}{u_{2}} d \eta^{\prime}
$$

and $u_{2}$ is the fluid velocity behind the detonation. Although Equation (3) does allow for varying density and viscosity through the boundary layer, we make the simplifying assumption that

$$
\frac{\rho \mu}{\rho_{2} \mu_{2}}=C=1
$$


where $C$ is the Chapman-Rubesin parameter. The boundary conditions are that of zero flow velocity at the wall and uniform velocity $u_{2}$ as $\eta \rightarrow \infty$

$$
\begin{aligned}
f(0) & =f^{\prime}(0)=0 \\
\lim _{\eta \rightarrow \infty} f^{\prime}(\eta) & =1 .
\end{aligned}
$$

Applying the boundary layer analysis to the conservation of energy equation results in

$$
0=\frac{1}{P r} g^{\prime \prime}+\frac{u_{2}^{2}}{h_{2}} f^{\prime \prime 2}+\frac{1}{2}\left(\eta-\frac{u_{2}}{U_{C J}} f\right) g^{\prime}
$$

where $\operatorname{Pr}$ is the Prandtl number and is assumed to equal $0.7, h$ is enthalpy, and, for a perfect gas,

$$
g(\eta)=\frac{h(\eta)}{h_{2}}=\frac{T(\eta)}{T_{2}} .
$$

The boundary conditions are given by the wall temperature and free-stream enthalpy

$$
\begin{aligned}
h(0) & =h_{w}=c_{p, 2} T_{w} \\
\lim _{\eta \rightarrow \infty} h(\eta) & =1 .
\end{aligned}
$$

Equations (9) and (10) use $h=c_{p, 2} T$ and assume that the specific heat is constant behind the detonation front and the effects of chemical reaction are negligible. In general, the flow within the boundary layer is reacting and non-equilibrium, which requires a numerical solution and the resulting flow field is not selfsimilar.

The solutions to Equations 3 and 8 were obtained using the Matlab function ode45 combined with a shooting technique to implement the boundary conditions. Once the temperature profile was known, the heat flux to the wall was computed using

$$
\dot{q}=\left.k \frac{d T}{d y}\right|_{y=0}
$$

where $k$ is the thermal conductivity in the fluid. This predicted heat flux is compared with the measured heat flux behind detonations in Section IV.

\section{B. Detonation Reflection}

A semi-empirical model has been developed ${ }^{4,25}$ in our laboratory that predicts the speed and strength of a reflected detonation. The relevant portion of the model is that the speed of the reflected detonation is given by

$$
U_{R}(t)=-u(x, t)+c(x, t) \sqrt{1+\frac{\gamma+1}{2 \gamma}\left[\frac{p_{R}(t)}{p(x, t)}-1\right]}
$$

where theoretical values for post-detonation fluid velocity $u$, sound speed $c$, pressure $p$, and ratio of specific heats $\gamma$ are calculated using Cantera $1.8^{26}$ and the pressure behind the shock is given by

$$
p_{R}(t)=\left(p_{C J, r e f}-p_{3}\right) \exp \left[-\frac{t-t_{0}}{\tau}\right]+p_{3}
$$

where $t_{0}$ is the time of reflection, $\tau$ is a time constant corresponding to the pressure decay caused by the TZ expansion, the final equilibrium pressure $p_{3}$ is calculated using Cantera and the theoretical peak reflected detonation pressure, $p_{C J, r e f}$ is calculated using the Shock and Detonation Toolbox. ${ }^{23}$ For a very long detonation tube, such as considered here, $\tau \gg t-t_{0}$ implying that $p_{R}$ may be approximated as

$$
p_{R}(t)=p_{C J, r e f}
$$


and

$$
U_{R}=U_{R, C J}
$$

which is also computed using the shock and detonation toolbox ${ }^{23}$ values for the present experiments are given in Table 1.

\section{Experimental Setup}

Experiments were performed in the GALCIT Detonation Tube (GDT) ${ }^{27}$ shown in Figure 4. The GDT is a $7.6 \mathrm{~m}$ long, inner diameter $280 \mathrm{~mm}$ detonation tube equipped with a test section of $150 \mathrm{~mm}$ wide square cross-section and two quartz windows to provide optical access. The tube was initially evacuated and then filled via the method of partial pressures to the desired composition. The experimental work presented herein is a stoichiometric hydrogen-oxygen mixture diluted with varying amounts of argon at varying initial pressure as given in Table 1. These run conditions were chosen to give a range of cell sizes and postdetonation temperatures allowing us to examine these effects on the wave reflection process. Increasing the initial pressure decreases the cell size from approximately $14 \mathrm{~mm}^{28}$ for an initial pressure of $10 \mathrm{kPa}$ to $3 \mathrm{~mm}$ for an initial pressure of $40 \mathrm{kPa}^{29}$ Argon dilution increases the cell size and decreases the post-detonation temperature.

The experiment was initiated by injecting a mixture of acetylene-oxygen into the ignition end of the GDT for a duration of $4.5 \mathrm{~s}$. This injection was followed by a $1 \mathrm{~s}$ settling period before a $2 \mu \mathrm{F}$ capacitor charged to $9 \mathrm{kV}$ discharged through an $80 \mu \mathrm{m}$ diameter copper wire located in the ignition end of the GDT. This vaporized the copper wire and created a blast wave in the acetylene-oxygen, thereby initiating a detonation. This wave propagated into the $\mathrm{H}_{2}-\mathrm{O}_{2}$ mixture where it decayed in speed to the Chapman-Jouguet speed.

After ignition, a detonation enters the test section shown in Figure 5(a). ${ }^{30}$ A splitter plate was constructed (see Figure 5(b)) that raised the location of the boundary layer into the center of the windows. This splitter plate was instrumented with 12 PCB 113B26 pressure transducers and 12 surface junction thermocouples identical to those employed by Sanderson and Sturtevant ${ }^{31}$ for measuring heat flux inside the test section. The thermocouples are connected to a TrikTek Model 205B instrumentation amplifier with a response time of $7.5 \mu \mathrm{s}$ to a unit step input. In comparing this response time to the signal response, it seems that the gauge response time is sufficiently small so that the amplifiers dominate the overall response time. The gauges themselves are hand made and may have considerable differences in response. Calibration efforts along the lines of Mohammed et al. ${ }^{32,33}$ would be necessary to better deduce the gauge response. The spectral method employed by Sanderson and Sturtevant ${ }^{31}$ for reducing the heat flux data is also employed here. The locations of all gauges are given on the all relevant plots.

A Z-type schlieren system is used to visualize the incident detonation and shock wave reflection. The schlieren system consists of a pulsed EverGreen 70 PIV laser with a pulse width duration less than or equal to $10 \mathrm{~ns}$ and a Cooke PCO.2000 digital 14 bit CCD camera system. This allows two images to be taken in quick succession and was used to visualize the incoming detonation and the reflected shock wave for each test.

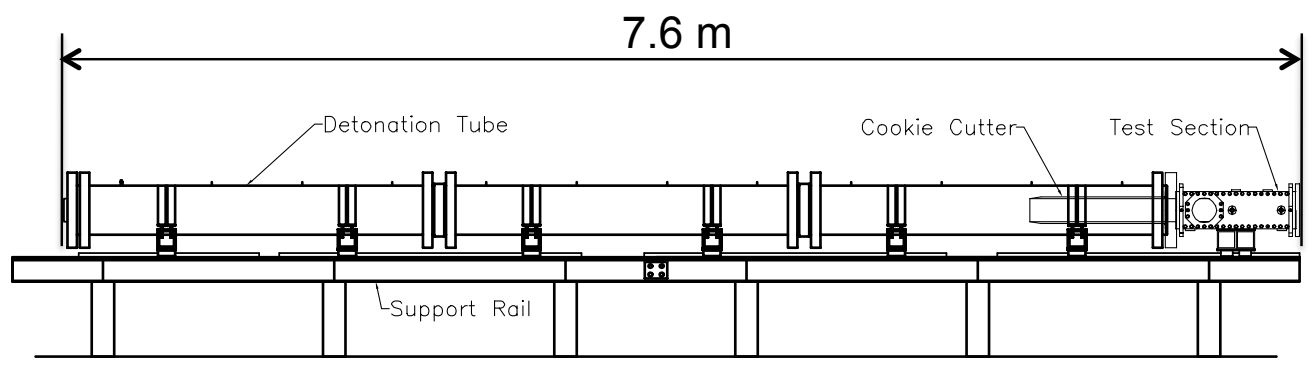

Figure 4. Schematic of the GALCIT Detonation Tube in which detonation experiments were performed (for more information, see Akbar $1997^{27}$ ). 


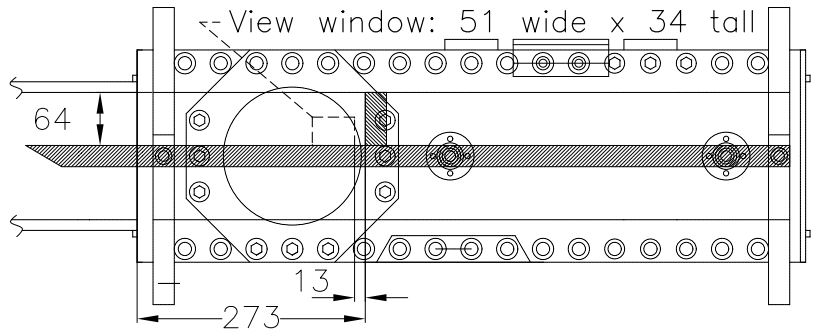

(a)

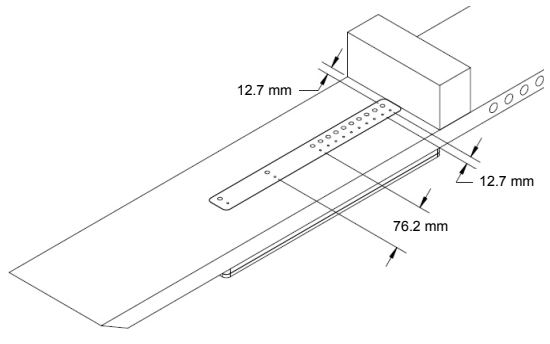

(b)

Figure 5. Detail views of the test section for the GDT; dimensions in $\mathrm{mm}$. (a) Location of viewing window relative to end-wall. (b) Pressure and thermocouple gauge locations relative to end-wall.

Table 1. Run conditions with measured incident shock speed $U_{I}$, theoretical CJ detonation speed $U_{C J}$, fluid velocity $u_{2}$, temperature behind the incident wave $T_{2}$, equilibrium ratio of specific heats $\gamma_{e, 2}$ behind the incident wave, ${ }^{34}$ unit Reynolds number, mean measured reflected shock speed over the first $127 \mathrm{~mm}$ Stof reflected wave propagation, and computed reflected shock speed.

\begin{tabular}{|c|c|c|c|c|c|c|c|c|c|}
\hline $\begin{array}{l}\text { Initial Pressure } \\
(\mathrm{kPa})\end{array}$ & Argon dilution & $\begin{array}{l}U_{I}^{\mathrm{a}} \\
(\mathrm{m} / \mathrm{s})\end{array}$ & $\begin{array}{l}U_{C J}{ }^{\mathrm{b}} \\
(\mathrm{m} / \mathrm{s})\end{array}$ & $\begin{array}{l}u_{2} \mathrm{~b} \\
(\mathrm{~m} / \mathrm{s})\end{array}$ & $\begin{array}{l}T_{2} \mathrm{~b} \\
(\mathrm{~K})\end{array}$ & $\gamma_{e, 2} \mathrm{~b}$ & $R e_{1}{ }^{\mathrm{c}}$ & $\begin{array}{l}\bar{U}_{R, \text { meas }}{ }^{\mathrm{a}} \\
(\mathrm{m} / \mathrm{s})\end{array}$ & $\begin{array}{l}U_{R, c o m p}{ }^{\mathrm{d}} \\
(\mathrm{m} / \mathrm{s})\end{array}$ \\
\hline \multirow[t]{3}{*}{10.0} & $0 \%$ & 2702 & 2719 & 1239 & 3272 & 1.12 & $0.49 \cdot 10^{6}$ & 1005 & 1026 \\
\hline & $50.0 \%$ & 1838 & 1854 & 827 & 3055 & 1.15 & $0.64 \cdot 10^{6}$ & 699 & 717 \\
\hline & $66.7 \%$ & 1681 & 1664 & 737 & 2892 & 1.18 & $0.66 \cdot 10^{6}$ & 688 & 668 \\
\hline \multirow[t]{4}{*}{25.0} & $0 \%$ & 2763 & 2760 & 1260 & 3425 & 1.12 & $1.20 \cdot 10^{6}$ & 1038 & 1049 \\
\hline & $50.0 \%$ & 1865 & 1872 & 840 & 3179 & 1.15 & $1.56 \cdot 10^{6}$ & 709 & 736 \\
\hline & $66.7 \%$ & 1684 & 1691 & 745 & 2992 & 1.19 & $1.62 \cdot 10^{6}$ & 686 & 686 \\
\hline & $83.3 \%$ & 1435 & 1457 & 589 & 2474 & 1.35 & $1.43 \cdot 10^{6}$ & 720 & 661 \\
\hline \multirow[t]{4}{*}{40.0} & $0 \%$ & 2792 & 2786 & 1270 & 3508 & 1.12 & $1.90 \cdot 10^{6}$ & 1033 & 1061 \\
\hline & $50.0 \%$ & 1888 & 1889 & 846 & 3245 & 1.16 & $2.47 \cdot 10^{6}$ & 728 & 746 \\
\hline & $66.7 \%$ & 1699 & 1705 & 750 & 3045 & 1.20 & $2.57 \cdot 10^{6}$ & 701 & 696 \\
\hline & $83.3 \%$ & 1445 & 1462 & 588 & 2489 & 1.37 & $2.25 \cdot 10^{6}$ & 720 & 670 \\
\hline
\end{tabular}

a Measured using time of arrival from PCB pressure transducers.

b Computed using Cantera $1.8^{26}$ and the Shock and Detonation Toolbox. ${ }^{23}$

c Computed using Equation (17) at time $t-t_{a}=1 \mathrm{~m} / U_{C J}$ corresponding to a location $1 \mathrm{~m}$ behind the detonation.

d Computed using the ideal reflection model described in the shock and detonation toolbox. ${ }^{23}$ 


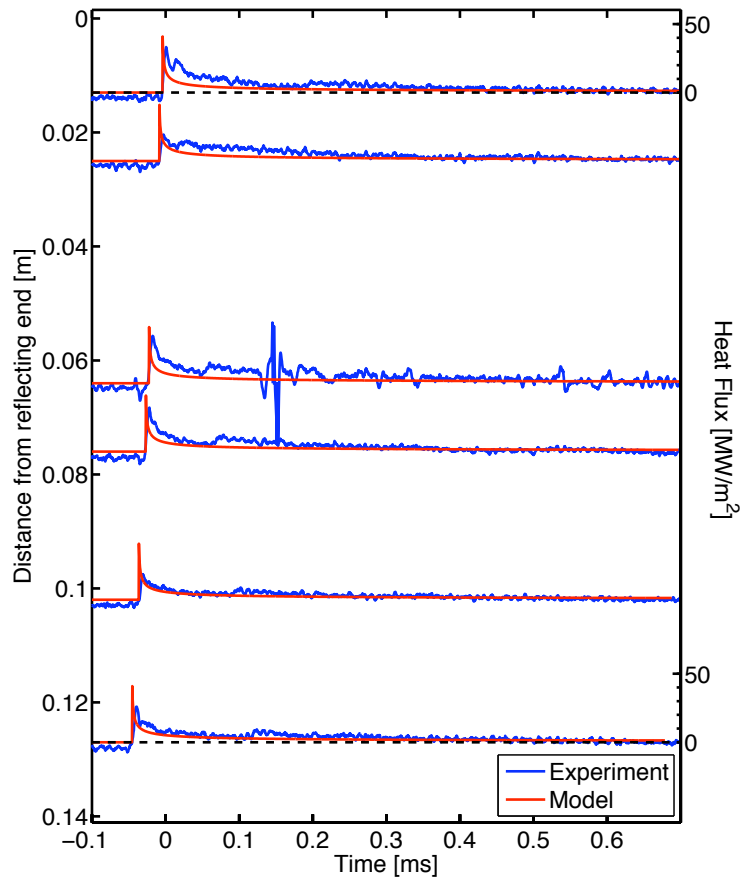

(a)

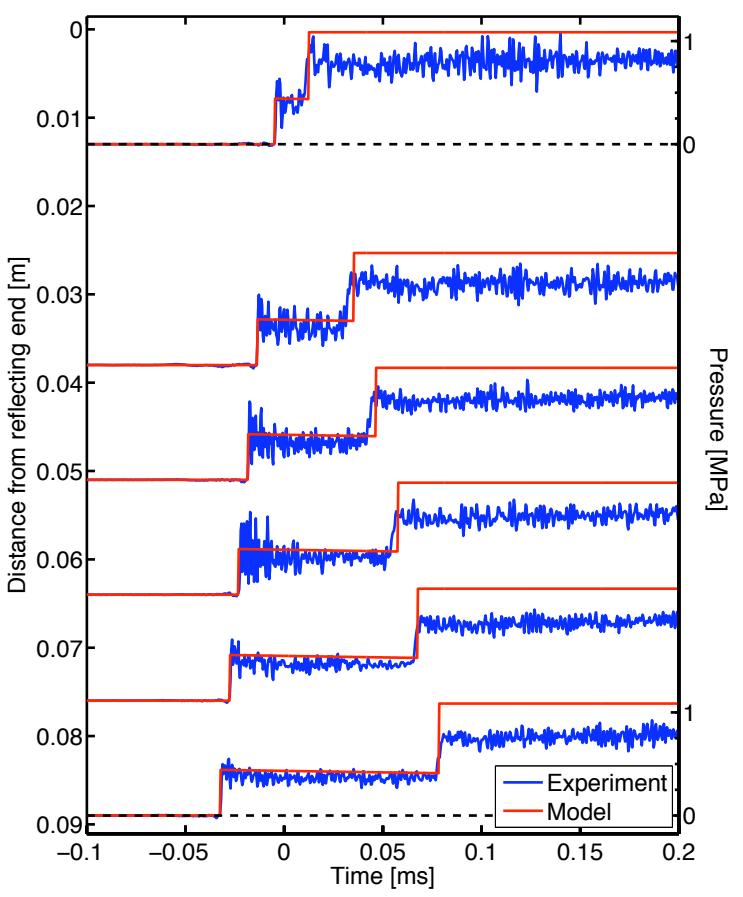

(b)

Figure 6. Results for a stoichiometric hydrogen-oxygen detonation of initial pressure $25 \mathrm{kPa}$ with $0 \%$ argon dilution. Experimental measurements (blue) are compared to (a) the laminar boundary layer heat flux model discussed in Section II A and (b) the one-dimensional pressure model discussed in Section II B and

\section{Discussion}

Figure 6 portrays results for $0 \%$ argon dilution; this case has the largest discrepancy between observed and computed reflected shock pressures of the cases examined in this paper. The heat flux plot in Figure 6(a) shows the heat transfer measurements compared to the laminar boundary layer theory described in Section II A. The incident detonation is clearly marked by a spike in both measured and predicted heat transfer. The reflected detonation is much less clear and plays only a lesser role in the heat flux measurements. The heat-flux model is only valid for times before the arrival of the reflected shock. Figure 7 plots the heat-flux data in a $S t-R e$ plot where the Stanton and Reynolds ${ }^{35}$ numbers are computed from

$$
\begin{aligned}
& R e=\frac{\rho_{2} u_{2}^{2}\left(t-t_{a}\right)}{\mu_{2}} \\
& S t=\frac{\dot{q}}{\left(h_{2}-h_{w}\right) \rho_{2} u_{2}}
\end{aligned}
$$

where $t_{a}$ is the arrival time of the detonation. We observe that for times after the response time of the gauge and before the arrival of the reflected shock, the laminar theory does an adequate job of predicting the measured heat transfer. This suggests that the boundary layer is in fact laminar for this duration. Using a turbulent transition Reynolds number of ${ }^{35} R e_{T} \approx 6 \cdot 10^{5}$ it is perhaps no surprise that transition does not occur before the arrival of the reflected shock (which corresponds to $R e_{r e f}=5.5 \cdot 10^{5}$ for the undiluted initial pressure $25 \mathrm{kPa}$ detonation). However a substantial difference between shock and detonation waves is the presence of transverse waves behind detonations. This work indicates that the transverse waves do not significantly effect turbulent transition for the mixtures tested. This agrees with the work of Liu et al. ${ }^{16}$ who found reasonable qualitative agreement with the experimental work of Laderman et al. ${ }^{36}$ with a laminar boundary layer model applied to hydrogen-oxygen mixtures of initial pressure $1 \mathrm{~atm}$. The best agreement with the experimental measurements comes from a power law curve fit from Hartunian ${ }^{37}$ as denoted by the 
subscript $H$ :

$$
S t_{H, l a m}=\frac{0.8}{\sqrt{R e}} \quad S t_{H, t u r b}=\frac{3.7 \cdot 10^{-2}}{R e^{1 / 5}} .
$$

This fit is also plotted on Figure 7.

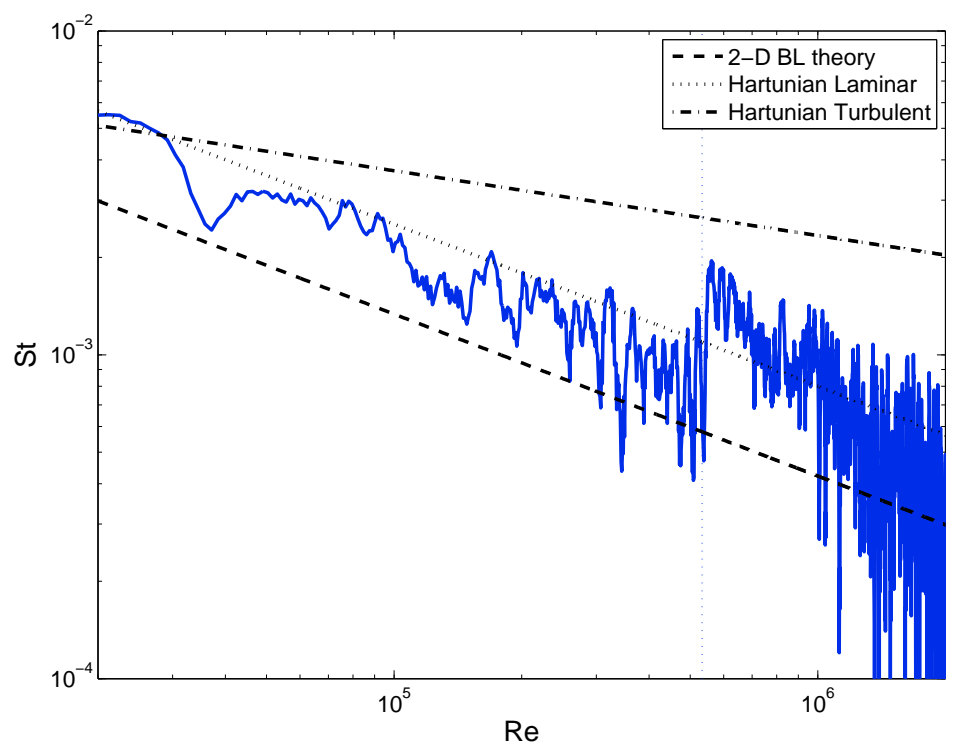

(a)

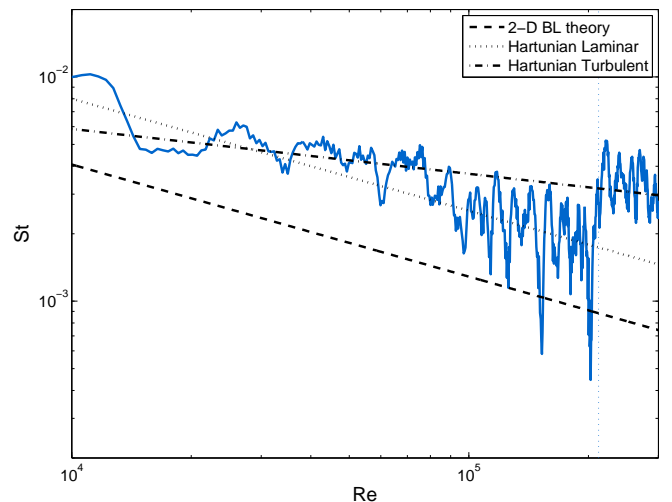

(b)

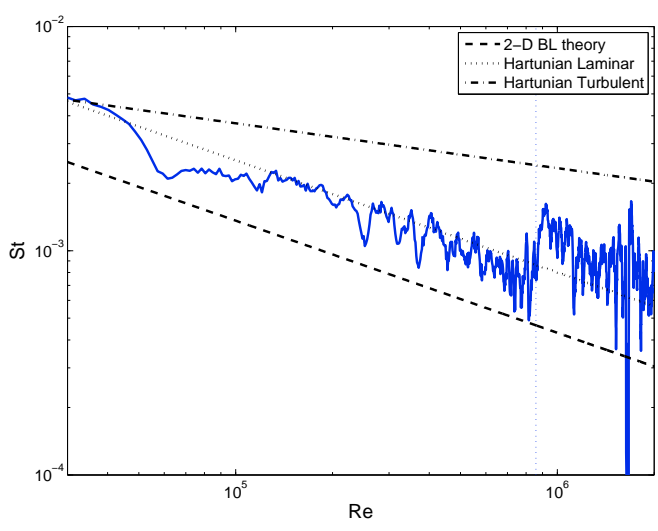

(c)

Figure 7. Stanton-Reynolds plot comparing measured and computed heat flux for a stoichiometric hydrogenoxygen detonation with $0 \%$ argon dilution at a distance of $127 \mathrm{~mm}$ from the reflecting end wall; initial pressure (a) $25 \mathrm{kPa}$, (b) $10 \mathrm{kPa}$, and (c) $40 \mathrm{kPa}$. The dashed black line indicates the arrival of the reflected wave.

The trend of agreement with the laminar boundary layer theory is seen for all initial pressures tested as observed in Figures 7(b), 7(c), and 8 with the largest differences occurring in the $10 \mathrm{kPa}$ initial pressure case where the Reynolds number is smaller and the cell size larger than the other considered cases. We also see on each heat flux graph that the heat transfer is under-predicted near the location of detonation reflection indicating the wave reflection process and resulting fluid dynamics are more complicated than incorporated into the simple two-dimensional laminar boundary layer theory.

Examining the corresponding pressure plot, Figure 6(b), we observe two sharp increases in pressure for each measurement location. The first increase is due to the incident detonation; following this rise are highfrequency pressure oscillations corresponding to the transverse waves associated with gaseous detonations. The second increase occurs from the reflected shock wave propagating back towards the location of ignition. Plotted with the experimental data are the predictions of the model introduced in Section II B. We see that the arrival time and resulting pressure of the incident detonation is well-predicted by the one-dimensional 


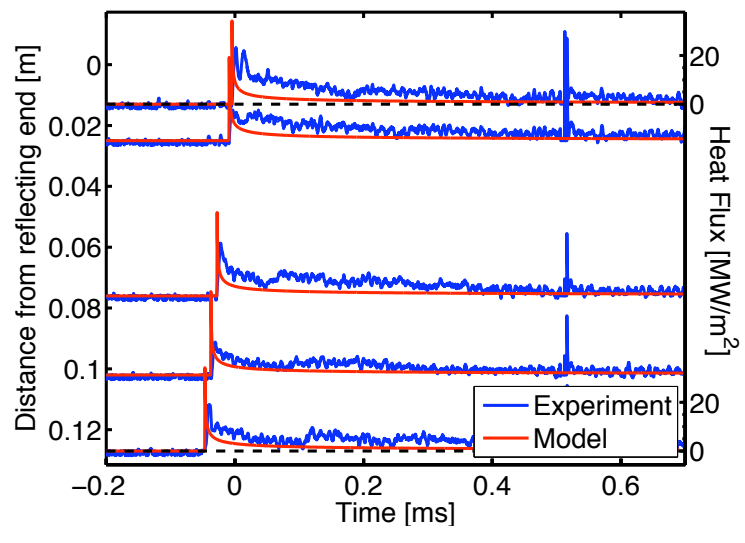

(a)

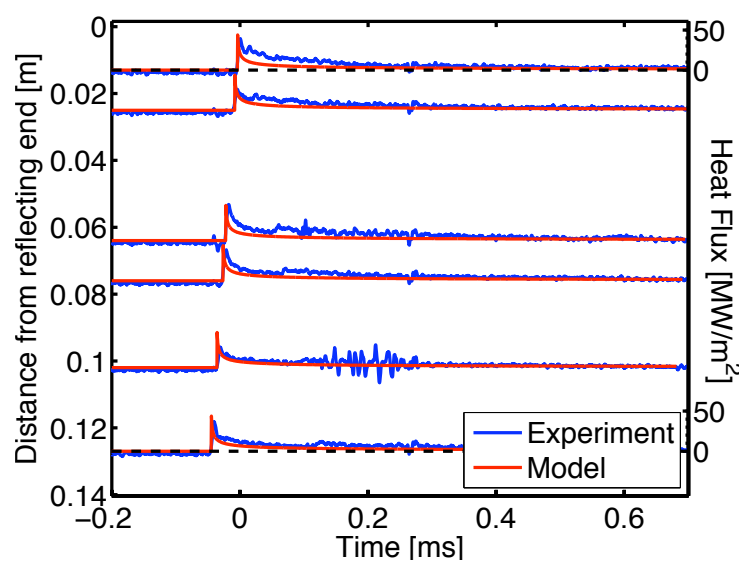

(b)

Figure 8. Heat flux measurements for detonations of stoichiometric hydrogen-oxygen with $0 \%$ argon dilution at initial pressure (a) $10 \mathrm{kPa}$ and (b) $40 \mathrm{kPa}$.

Chapman-Jouguet theory. The reflected shock wave, however, is not adequately described by the pressure model. The reflected shock speed is within $8 \%$ of the predicted value in the highest dilution case and $3 \%$ for the undiluted case. However the reflected shock pressure is under predicted by up to $40 \%$ in the undiluted cases and up to $6 \%$ in the argon dilution cases. This trend of accurate arrival time, but inaccurate pressures is the same as observed in earlier work ${ }^{4}$ and initially suggested the possibility of shock wave-boundary layer interaction. Figure 9 shows the schlieren image corresponding to the graphs plotted in Figure 6 with Figure 9(a) showing the incident detonation propagating to the right and Figure 9(b) showing the reflected shock wave propagating back to the left. As is observed in the schlieren photographs, there is no significant shock wave-boundary layer interaction.

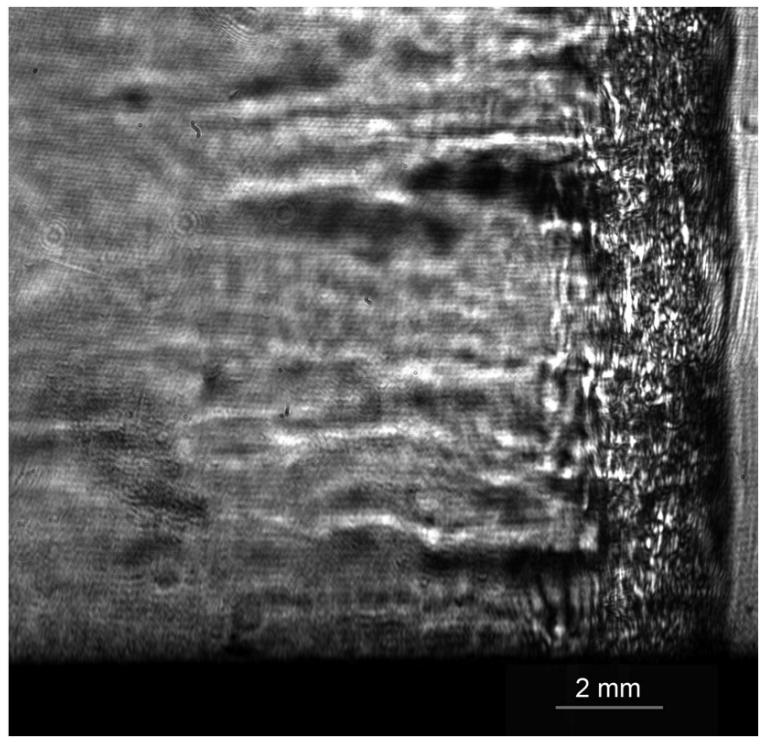

(a)

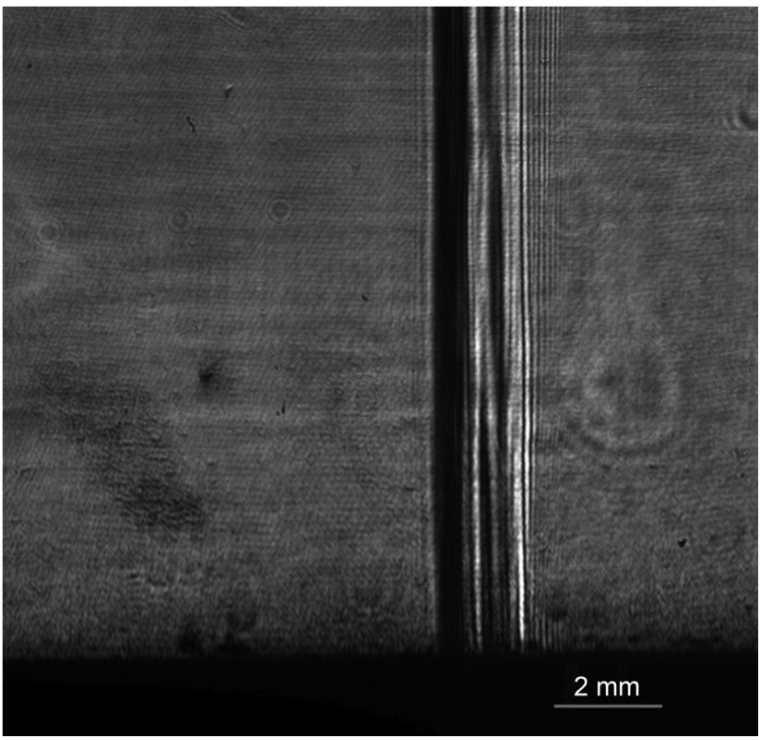

(b)

Figure 9. Schlieren images showing (a) incident detonation and (b) reflected shock wave for a stoichiometric hydrogen-oxygen detonation with no argon dilution at initial pressure $25 \mathrm{kPa}$.

Another feature of note in the pressure signals is that the rise time of the pressure signals through the reflected shock wave occurs over several microseconds-slower than would be expected from a shock. This corresponds to the reflected shock wave being apparently composed of multiple shocks as observed in Figures 9 and 10. The source of these shocks is not fully understood. The spacing does not seem to be a 
strong function of cell size as observed by essentially no change in the wave thickness for initial pressures of $10 \mathrm{kPa}$ (approximately $14 \mathrm{~mm}$ cell width ${ }^{28}$ ) and $40 \mathrm{kPa}$ (approximately $3 \mathrm{~mm}$ cell width ${ }^{29}$ ) as seen in Figure 10 .

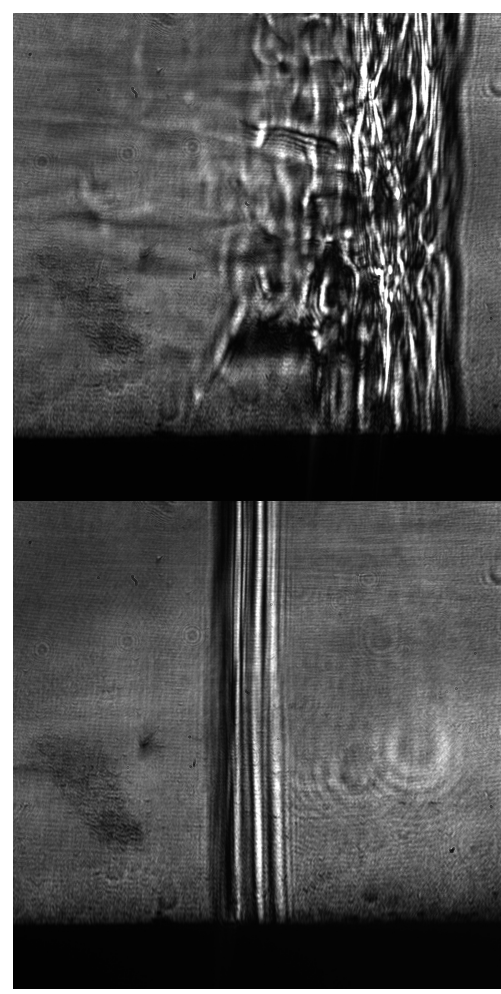

(a)

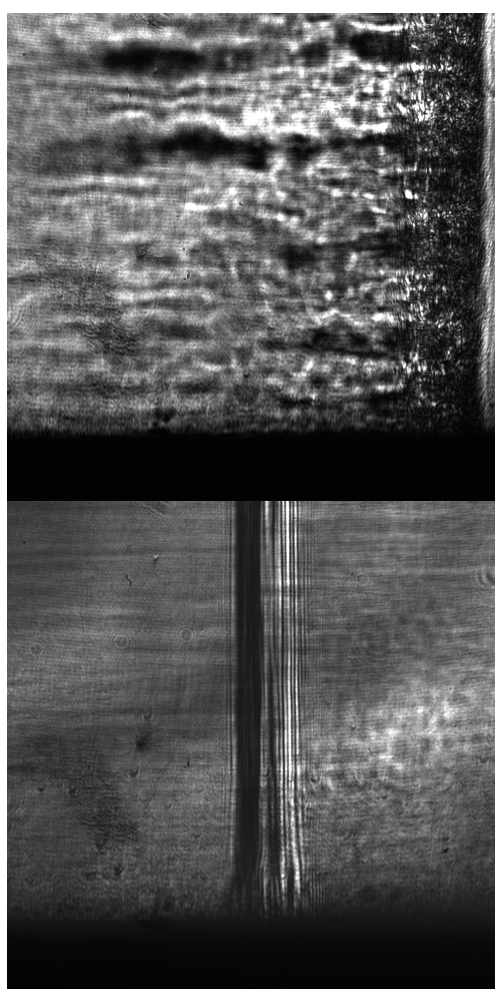

(b)

Figure 10. Schlieren images for stoichiometric hydrogen-oxygen detonations with no argon dilution at initial pressure (a) $10 \mathrm{kPa}$ and (b) $40 \mathrm{kPa}$.

We next examine the effects of argon dilution. Pressure traces for detonations in hydrogen-oxygen-argon at $25 \mathrm{kPa}$ and argon dilutions of $50,66.7$, and $83.3 \%$ is given in Figure 11 . We observe that the pressure signals are much better predicted by the analytical pressure model. This result is still under investigation; one significant point is that the effect of adding argon is to decrease the post-detonation temperature. The effect of the transverse waves on the wall pressure are clearly observed in the $83 \%$ argon dilution case shown in Figure 11(c) as additional pressure waves behind the incident detonation and in a decrease in the frequency of the pressure signals after the arrival of the detonation.

The schlieren images corresponding to the pressure measurements of Figure 11 are shown in Figure 12. Here we observe the general trend of the reflected shock wave becoming gradually thicker from $1.9 \mathrm{~mm}$ at $0 \%$ argon dilution to $2.6 \mathrm{~mm}$ at $83.3 \%$ argon dilution. We also observe a small amount of shock wave-boundary layer interaction for the 50 and $66.7 \%$ argon dilution cases as marked by the shock wave angling to the left near the floor of the detonation tube. This effect was unexpected due to the fact that argon is known ${ }^{9}$ to inhibit boundary layer interaction in shock tubes due to the increase in $\gamma$; however there is no visible boundary layer interaction in the $83.3 \%$ argon dilution case.

Although the measurements and schlieren images were not shown for the remaining test cases, similar qualitative trends were observed in both pressure measurements and schlieren images. The largest difference in the remaining data is that the heat flux measurements became less useful at higher dilutions and lower pressures due to the decreased signal amplitude resulting in an unfavorable signal-to-noise ratio.

\section{Conclusions}

The heat flux to the tube wall and wall pressure are investigated behind stoichiometric hydrogen-oxygen detonations. Laminar boundary layer theory well-predicts the heat transfer observed behind gaseous deto- 


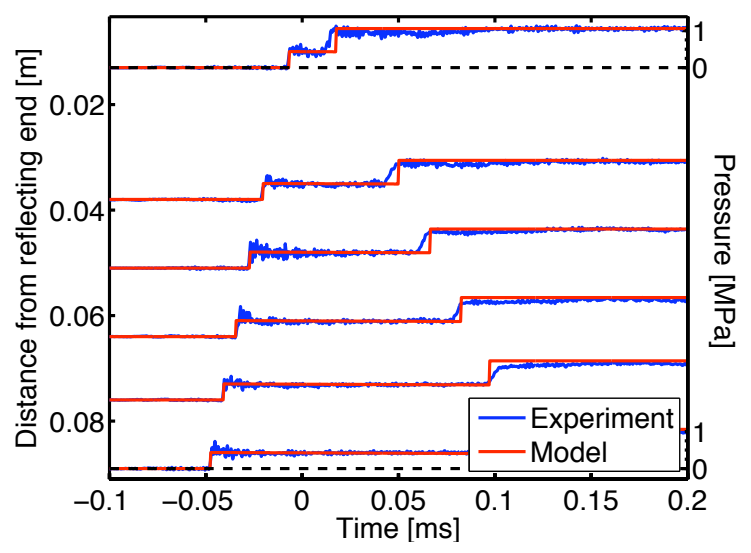

(a)

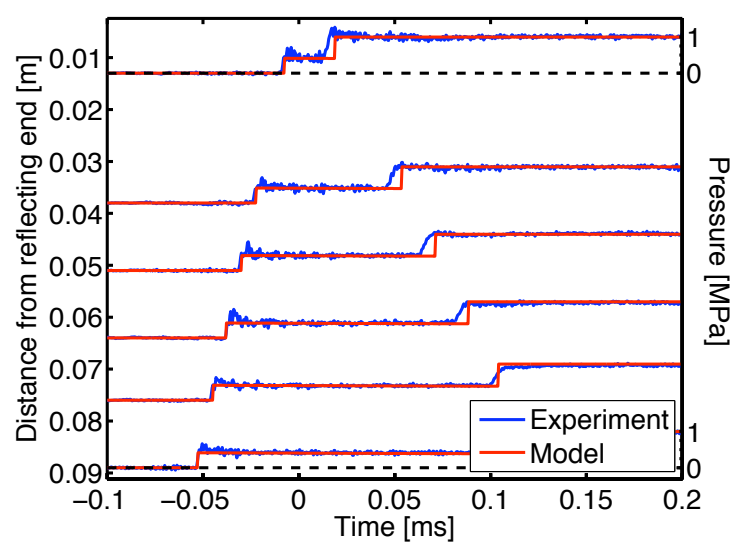

(b)

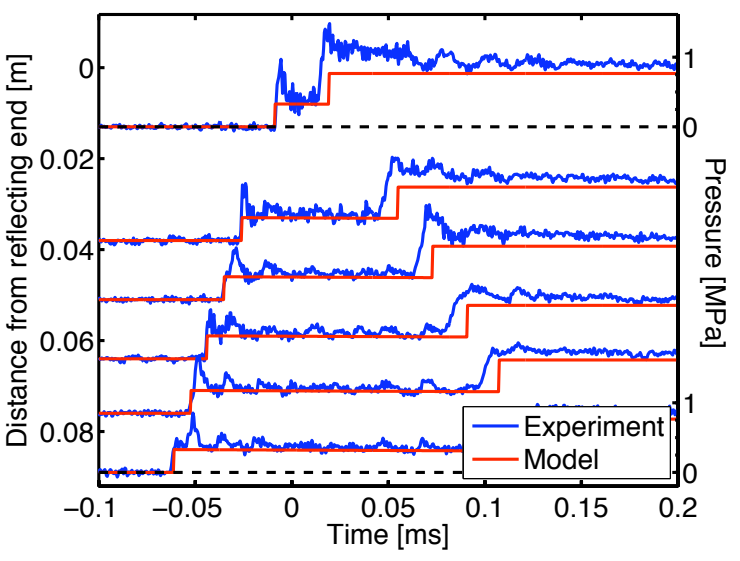

(c)

Figure 11. Pressure measurements for detonations of stoichiometric hydrogen-oxygen at initial pressure $25 \mathrm{kPa}$ with (a) $50 \%$, (b) $66.7 \%$, and (c) $83.3 \%$ argon dilution. 


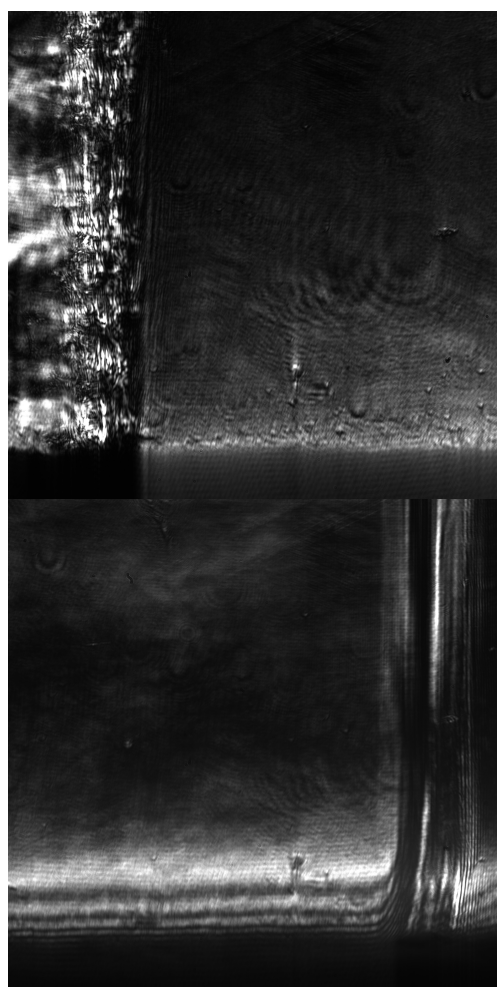

(a)

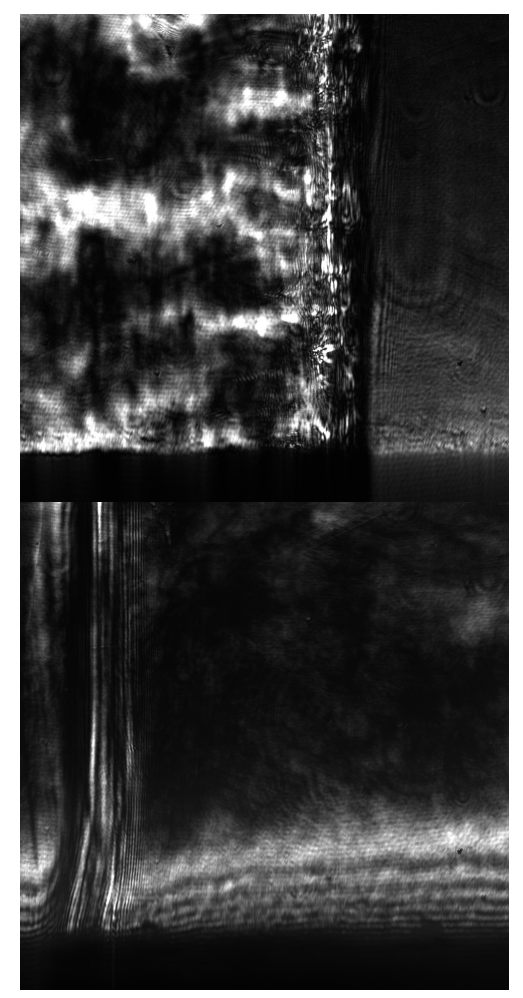

(b)

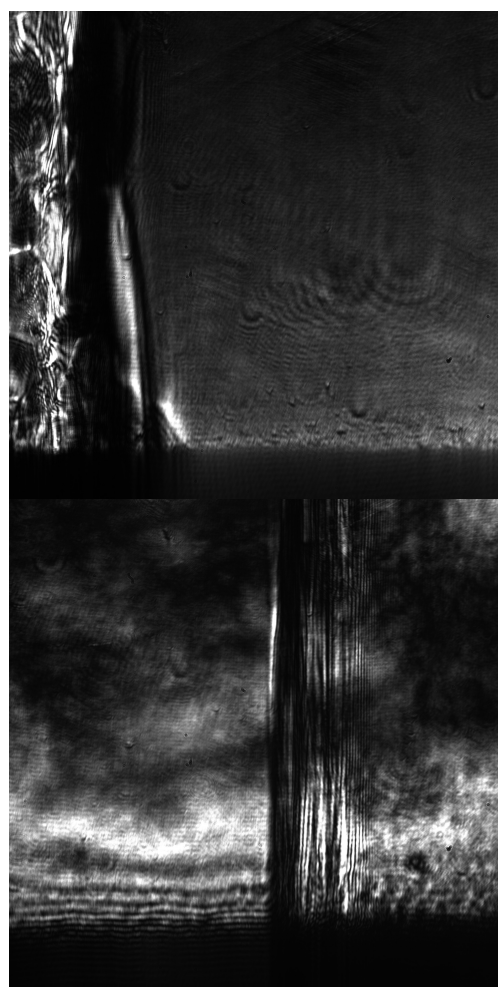

(c)

Figure 12. Schlieren images of stoichiometric hydrogen-oxygen detonations of initial pressure $25 \mathrm{kPa}$ with (a) $50 \%$, (b) $66.7 \%$, and (c) $83.3 \%$ argon dilution.

nations for the $25 \mathrm{kPa}$ and $40 \mathrm{kPa}$ initial pressure cases. This suggests that the boundary layer for these cases is laminar. The laminar theory is less accurate for the $10 \mathrm{kPa}$ initial pressure case; the source of this discrepancy is still under investigation.

The behavior of the reflected shock wave created when a detonation normally impinges upon an end wall is investigated. The ideal model of detonation reflection predicts the speed of the reflected wave within 3-8\%. The pressure behind the reflected waves is substantially under predicted (up to 40\%) for the undiluted cases, but the agreement is much better (within 6\%) for the diluted cases. This trend was previously observed in the work of Karnesky et al. ${ }^{4}$ and was thought to be caused by shock wave-boundary layer interaction. The present work, however, discounts such interaction as the source of the discrepancy through schlieren photographs taken of the reflected shock wave. Paradoxically, some degree of interaction is observed for argon dilutions of 50 and $67 \%$ although the ideal model more accurately predicts the pressure behind the reflected wave. These findings are puzzling and still under investigation.

\section{Acknowledgments}

This research is sponsored by the DHS through the University of Rhode Island, Center of Excellence for Explosives Detection. The authors would also like to thank Bahram Valiferdowsi for his help in designing the splitter plate as well as the Caltech SURF program for sponsoring Jeff Odell.

\section{References}

${ }^{1}$ Shepherd, J. E., "Structural Response of Piping to Internal Gas Detonation," Journal of Pressure Vessel Technology, Vol. 131, No. 3, June 2009, pp. 87-115.

${ }^{2}$ Lee, J. H. S., The Detonation Phenomenon, Cambridge University Press, 2008.

${ }^{3}$ Fickett, W. and Davis, W. C., Detonation, University of California Press, Berkerely, CA, 1979.

${ }^{4}$ Karnesky, J., Damazo, J., Shepherd, J. E., and Rusinek, A., "Plastic response of thin-walled tubes to detonation," ASME 2010 Pressure Vessels and Piping Conference., ASME, 2010. 
${ }^{5}$ Shepherd, J., Teodorcyzk, A., Knystautas, R., and Lee, J., "Shock Waves Produced by Reflected Detonations," Progress in Astronautics and Aeronautics, Vol. 134, 1991, pp. 244-264.

${ }^{6}$ Taylor, G. I., "The Dynamics of the Combustion Products behind Plane and Spherical Detonation Fronts in Explosives," Proc. Roy. Soc., Vol. A200, 1950, pp. 235-247.

${ }^{7}$ Zel'dovich, Y. B., "On the Theory of the Propagation of Detonations in Gaseous Systems," JETP, Vol. 10, 1940, pp. 542568, Available in translation as NACA TM 1261 (1950).

${ }^{8}$ Damazo, J., Ziegler, J., Karnesky, J., and Shepherd, J. E., "Investigating Shock Wave-Boundary Layer Interaction Caused by Reflecting Detonations," Proceedings of the $8^{\text {th }}$ ISPHMIE Conference, Yokohama, Japan, September 5-10 2010.

${ }^{9}$ Mark, H., "The Interaction of a Reflected Shock Wave with the Boundary Layer in a Shock Tube," Tech. Rep. TM1418, National Advisory Committee for Aeronautics, March 1958.

${ }^{10}$ Strehlow, R. A. and Cohen, A., "Limitations of the Reflected Shock Technique for Studying Fast Chemical Reactions and Its Application to the Observation of Relaxation in Nitrogen and Oxygen," Journal of Chemical Physics, Vol. 30, No. 1, 1959 , pp. $257-265$.

${ }^{11}$ Taylor, J. R. and Hornung, H. G., "Real Gas and Wall Roughness Effects on the Bifurcation of the Shock Reflected from the End Wall of a Tube," Proceedings of the $13^{\text {th }}$ International Symposium on Shock Tubes and Waves, Niagara Falls, USA, July 6-9 1981.

${ }^{12}$ Petersen, E. L. and Hanson, R. K., "Improved Turbulent Boundary-Layer Model for Shock Tubes," AIAA Journal, Vol. 41, No. 7, 2003, pp. 1314-1322.

${ }^{13}$ Mirels, H., "Boundary Layer Behind Shock or Thin Expansion Wave Moving Into Stationary Fluid," NACA TN 3712 , Lewis Flight Propulsion Laboratory, Cleveland, Ohio, 1956.

${ }^{14}$ Mirels, H., "Laminar Boundary Layer Behind a Strong Shock Moving Into Air," NACA TN D-291, Lewis Flight Propulsion Laboratory, Cleveland, Ohio, 1961.

${ }^{15}$ Sturtevant, B. and Okamura, T. T., "Dependence of Shock-Tube Boundary Layers on Shock Strength," The Physics of Fluids, Vol. 12, No. 8, 1969, pp. 1723-1725.

${ }^{16} \mathrm{Liu}$, W. S., Du, X. X., and Glass, I. I., "Laminar Boundary Layers Behind Detonation Waves," Proceedings of the Royal Society of London Series A, Mathematical and Physical Sciences, Vol. 387, No. 1793, June 1983, pp. 331-349.

${ }^{17}$ Ziegler, J., Simulations of Compressible, Diffusive, Reactive Flows with Detailed Chemistry Using a High-Order Hybrid WENO-CD Scheme, Ph.D. thesis, California Institute of Technology, 2011.

${ }^{18}$ Gamezo, V. N., Khokhlov, A. M., and Oran, E. S., "The Influence of Shock Bifurcations on Shock-Flame Interactions and DDT," Combustion and Flame, Vol. 126, 2001, pp. 1810-1826.

${ }^{19}$ Weber, J., Oran, E., Boris, J., and Anderson, J., "The numerical simulation of shock bifurcation near the end wall of a shock tube," Physics of Fluids, Vol. 7, No. 10, 2006, pp. 2475-2488.

${ }^{20}$ Kessler, D., Gamezo, V., and Oran, E., "Three-dimensional reactive shock bifurcations," Proceedings of the Combustion Institute, Vol. 30, 2005, pp. 1841-1847.

${ }^{21}$ Davies, L. and Wilson, J. L., "Influence of Reflected Shock and Boundary-Layer Interaction on Shock-Tube Flows," Shock Tube Symposium, 1969.

${ }^{22}$ Nettleton, M. A., Gaseous Detonations, Chapman and Hall, NY, 1987.

${ }^{23}$ Browne, S., Zeigler, J., and Shepherd, J., "Numerical Solution Methods for Shock and Detonation Jump Conditions," Tech. Rep. FM2006-006, Graduate Aeronautical Laboratories California Institute of Technology, February 2008.

${ }^{24}$ Schlichting, H. and Gersten, K., Boundary-layer Theory, Springer, Berlin, 1965.

${ }^{25}$ Karnesky, J., Detonation Induced Strain in Tubes, PhD dissertation, California Institute of Technology, 2010.

${ }^{26}$ Goodwin, D. G., "Cantera - An open-source, extensible software suite for CVD process simulations," Electrochemical Society, 2003, pp. 155-162.

${ }^{27}$ Akbar, R., Mach Reflection of Gaseous Detonations, Ph.D. thesis, Rensselaer Polytechnic Institute, 1997.

${ }^{28}$ Strehlow, R., "Transverse waves in detonations: II. structure and spacing in $\mathrm{H}_{2}-\mathrm{O}_{2}, \mathrm{C}_{2} \mathrm{H}_{2}-\mathrm{O}_{2}, \mathrm{C}_{2} \mathrm{H}_{4}-\mathrm{O}_{2}$ and $\mathrm{CH}_{4}-\mathrm{O}_{2}$ systems," AIAA J, Vol. 7, 1969, pp. 492-496.

${ }^{29}$ Desbordes, D., Aspects stationnaires et transitoires de la detonation dans les gaz: relation avec la structure cellulaire du front, $\mathrm{PhD}$ dissertation, Universite de Poitiers, 1990.

${ }^{30}$ Kaneshige, M. J., Gaseous Detonation Initiation and Stabilization by Hypervelocity Projectiles, PhD dissertation, California Institute of Technology, 1999.

${ }^{31}$ Sanderson, S. R. and Sturtevant, B., "Transient Heat Flux Measurement Using a Surface Junction Thermocouple," Review of Scientific Instruments, Vol. 73, No. 7, 2002, pp. 2781-7.

${ }^{32}$ Mohammed, H., Salleh, H., and Yusoff, M., "Determination of the Effusivity of Different Scratched Coaxial Temperature Sensors Under Hypersonic Flow," International Journal of Thermophysics, Vol. 31, 2010, pp. 2305-2322.

${ }^{33}$ Mohammed, H., Salleh, H., and Yusoff, M., "The Effect of Scratch Technique on the Thermal-Product Value of Temperature Sensors," Thermophysics and Aeromechanics, Vol. 18, No. 1, 2011, pp. 51-64.

${ }^{34}$ Radulescu, M. I. and Hanson, R. K., "Effect of heat loss on pulse-detonation-engine flow fields and performance," Journal Of Propulsion And Power, Vol. 21, No. 2, Mar-Apr 2005, pp. 274-285.

${ }^{35}$ Petersen, E. L. and Hanson, R. K., "Measurement of reflected-shock bifurcation over a wide range of gas composition and pressure," Shock Waves, Vol. 15, 2006, pp. 333-340.

${ }^{36}$ Laderman, A., Hecht, G., and Oppenheim, A., Temperature - its measurement and control in science and industry, Reinhold Publishing Corporation, New York, 1962.

${ }^{37}$ Hartunian, R., Russo, A., and Marrone, P., "Boundary-Layer Transition and Heat Transfer in Shock Tubes," J. Aero. Sci, Vol. 27, 1960, pp. 587-594. 